\title{
Éclosion de tuberculose chez les consommateurs de drogues et les sans-abri du Grand Montréal, Canada, de 2003 à 2016
}

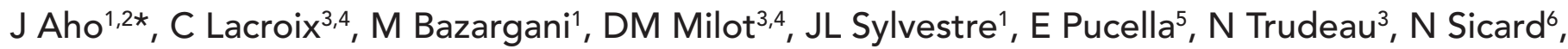 \\ N Savard ${ }^{1}, 7$, P Rivest $^{1}$, H Soualhine $^{8}$, M Munoz-Bertrand ${ }^{1}$
}

\section{Résumé}

Contexte : Au Canada, la tuberculose active est principalement diagnostiquée chez les migrants provenant de pays où la maladie est endémique et au sein des populations autochtones. Toutefois, des cas de tuberculose active chez les consommateurs de drogues et les sans-abri ont été déclarés dans le Grand Montréal depuis 2003.

Objectif : Décrire l'éclosion de tuberculose à Montréal selon les caractéristiques sociodémographiques, les facteurs de risque et les caractéristiques cliniques des cas, ainsi que l'intensité des interventions de santé publique, le suivi et la localisation des lieux de transmission potentielle.

Méthodologie : Tous les cas de tuberculose active ayant le génotype d'intérêt et résidant au Québec ainsi que les cas ayant un lien épidémiologique avec ces derniers ont été inclus dans l'investigation. Les données ont été extraites de façon rétrospective des enquêtes épidémiologiques de routine effectuées par les autorités de santé publique. Les caractéristiques des cas ont été décrites à l'aide du logiciel Excel. Une analyse spatiale des lieux fréquentés pendant les périodes de contagiosité des cas a été effectuée.

Résultats : Entre janvier 2003 et février 2016, 35 cas ont été recensés. La plupart des cas (86\%) étaient des non-Autochtones nés au Canada. De ce nombre, 28 cas présentaient plusieurs facteurs de risque, notamment la consommation de drogues (93\%), la consommation excessive d'alcool (64\%), l'itinérance (46\%), des comorbidités, telles qu'une co-infection VIH (36\%), et un stade avancé de la maladie. Sept cas ne présentant aucun facteur de risque étaient tous des contacts étroits de cas avec des facteurs de risque. L'intensité de la prise en charge des cas par les autorités de santé publique était élevée. Les lieux fréquentés par les cas présentant des facteurs de risque comprenaient des fumeries de crack, des refuges et des centres de réadaptation en dépendance dans le centre-ville de Montréal ainsi qu'un milieu résidentiel situé dans une région suburbaine.

Conclusion : Des éclosions de tuberculose peuvent se produire au sein de populations urbaines marginalisées nées au Canada, en particulier chez les personnes qui consomment des drogues. Des interventions adaptées à cette population sont nécessaires pour le dépistage et le diagnostic précoce de la tuberculose latente et de la tuberculose active de même que pour l'amélioration de la liaison avec les services de santé.

Citation proposée : Aho J, Lacroix C, Bazargani M, Milot DM, Sylvestre JL, Pucella E, Trudeau N, Sicard N, Savard N, Rivest P, Soualhine H, Munoz-Bertrand M. Éclosion de tuberculose chez les consommateurs de drogues et les sans-abri du Grand Montréal, Canada, de 2003 à 2016. Relevé des maladies transmissibles au Canada. 2017;43(3):79-84. https://doi.org/10.14745/ccdr.v43i34a03f

\section{Affiliations}

${ }^{1}$ Direction régionale de santé publique du Centre intégré universitaire de santé et de services sociaux du Centre-Sud-de-l'Île-de-Montréal, Montréal (Québec)

${ }^{2}$ Programme canadien d'épidémiologie de terrain, Agence de la santé publique du Canada, Ottawa (Ontario)

${ }^{3}$ Direction de santé publique du Centre intégré de santé et de services sociaux de la Montérégie-Centre, Longueuil (Québec)

${ }^{4}$ Faculté de médecine et des sciences de la santé, Université de Sherbrooke, Sherbrooke (Québec)

${ }^{5}$ Direction de santé publique du Centre intégré de santé et de services sociaux de Laval, Laval (Québec)

${ }^{6}$ Direction de la protection de la santé publique, Ministère de la Santé et des Services sociaux du Québec, Montréal (Québec)

7 Département d'épidémiologie, biostatistique et santé au travail, Université McGill, Montréal (Québec)

${ }^{8}$ Laboratoire de santé publique du Québec,

Sainte-Anne-de-Bellevue (Québec)

*Correspondance : josephine. aho.ccsmtl@ssss.gouv.qc.ca

\section{Introduction}

La tuberculose est une importante cause de morbidité et de mortalité dans le monde. En 2015, on estimait à 10,4 millions le nombre de nouveaux cas de tuberculose active et à 1,8 million le nombre de décès liés à la tuberculose (1). Le Canada est l'un des pays où l'incidence de la tuberculose est faible, atteignant son niveau historique le plus bas à 4,4/100 000 en 2014 (2,3). Comme dans la plupart des pays où l'incidence est faible, la tuberculose est concentrée dans des groupes précis - principalement au sein de populations autochtones et chez les migrants en provenance de pays où la maladie est endémique. Toutefois, des éclosions ont été déclarées à Vancouver, à Toronto, à Edmonton et à Ottawa ainsi que dans plusieurs villes des États-Unis, 
généralement au sein de groupes présentant des facteurs de risque, tels qu'une co-infection VIH (sida), I'itinérance, la consommation abusive d'alcool ou de drogues et l'incarcération (2-11). Bien que les données sur les taux d'incidence de tuberculose active au sein de ces groupes sont rares, un taux d'incidence de 13,2/100 000 personnes-années a été rapporté chez les sans-abri à Montréal (7).

Avec un taux d'incidence de tuberculose active de 2,9/100 000 en 2014, le Québec a la plus faible incidence de tuberculose au pays, après les provinces de l'Atlantique (3). Entre 2008 et 2011, $62,7 \%$ des cas ont été recensés parmi des migrants, 25,3\% parmi des non-Autochtones nés au Canada et 10,1\% parmi des Autochtones nés au Canada (12). La plupart des cas ont été recensés dans la région du Grand Montréal (12).

Tous les cas de tuberculose active sont à déclaration obligatoire au Québec et le traitement de la tuberculose active est obligatoire, les autorités de santé publique régionales ayant la capacité de prendre des mesures juridiques pour assurer l'observance du traitement (13). Lorsqu'un cas est déclaré par un médecin ou un laboratoire, la santé publique lance une enquête pour retracer et dépister les contacts du cas et assure le suivi du patient jusqu'à ce que le traitement soit terminé.

En 2003, quelques cas de tuberculose active liés à la même organisation communautaire ont été déclarés à Montréal. Plus tard, d'autres patients atteints de tuberculose active et provenant de quartiers défavorisés de Montréal et de régions avoisinantes ont été identifiés. Ils présentaient le même génotype et des facteurs de risques semblables, comme la consommation de drogues et l'itinérance. Cette constatation a marqué le début d'une éclosion qui se poursuit.

L'objectif du présent article est de décrire l'éclosion de tuberculose selon les caractéristiques sociodémographiques, les facteurs de risque et les caractéristiques cliniques des cas, ainsi que l'intensité des interventions de santé publique, le suivi et la localisation des lieux de transmission potentielle.

\section{Méthodologie}

\section{Identification des cas}

Dans cette étude rétrospective populationnelle, nous avons inclus tous les cas de tuberculose active confirmée avec le génotype d'intérêt qui résidaient dans la province du Québec au moment du diagnostic et tous les cas probables ayant un lien épidémiologique avec des cas confirmés. Les cas confirmés ont été identifiés au moyen du génotypage par la méthode des unités répétitives dispersées sur le génome mycobactérien (MIRU-VNTR) ou par la méthode du polymorphisme de longueur des fragments de restriction (RFLP). Le génotype d'intérêt correspondait à un profil RFLP à 11 bandes, à un profil MIRU à 12-loci ou à un profil MIRU à 24-loci après 2006, lorsque cette technologie était accessible. Ces profils RFLP, MIRU 12-loci et à 24-loci définissent la même souche. Toutes les souches de Mycobacterium tuberculosis ont été mises en culture et identifiées au Laboratoire de santé publique du Québec (LSPQ). Le génotypage par profil MIRU à 24-loci (14) est effectué systématiquement par le Laboratoire national de microbiologie dans le cadre d'un projet de collaboration avec le LSPQ ayant cours depuis 2012 (bien que le génotypage ait été effectué au cas par cas avant 2012).

\section{Collecte de données}

Chaque direction régionale de santé publique a extrait les données recueillies dans le cadre de leurs enquêtes de routine sur les cas et de leur suivi. Les données anonymes de Montréal, de Laval et de la Montérégie ont été compilées afin d'être analysées par la Direction régionale de santé publique du Centre intégré universitaire de santé et de services sociaux du Centre-Sud-de-l'Île-de-Montréal (Direction de santé publique de Montréal).

Les variables d'intérêt comprenaient les caractéristiques sociodémographiques, les facteurs de risque, les caractéristiques cliniques, le suivi et l'intensité des interventions de santé publique ainsi que les endroits visités pendant la période de contagiosité. Les renseignements sur les facteurs de risque à l'étude, notamment consommation de drogues, consommation d'alcool, tabagisme, travail du sexe, itinérance, incarcération au cours des deux dernières années, comorbidités telles qu'antécédents de tuberculose active, co-infection $\mathrm{VIH}$ ou hépatite $\mathrm{C}$ et troubles de santé mentale, ont été systématiquement recueillis à partir de l'enquête de la santé publique. Les caractéristiques cliniques comprenaient le site d'infection, les symptômes, les résultats des tests (radiographie du thorax et frottis positifs), I'hospitalisation, le traitement et l'évolution clinique. Les variables associées à l'intervention et au suivi par la santé publique comprenaient le recours à des mesures juridiques, comme les ordres d'isolement ou les ordonnances de cour, ainsi que l'intensité de l'intervention des autorités de santé publique. L'intensité moyenne des interventions de santé publique menées pendant toute la durée du suivi a été évaluée à l'aide d'une échelle ordinale établie avec des infirmières de santé publique. L'échelle était graduée en fonction du nombre moyen d'interventions (comme les appels téléphoniques et les visites à des patients ou à un professionnel de la santé concerné par le cas) qui ont été nécessaires pour mener l'enquête et pour assurer l'isolement du patient lorsque nécessaire et l'observance du traitement. L'échelle était répartie sur quatre niveaux d'intervention : faible (moins d'une intervention toutes les 4 à 6 semaines); normal (une intervention toutes les 4 à 6 semaines); élevé (une intervention toutes les 2 à 3 semaines); et très élevé (une intervention ou plus par semaine).

Les endroits visités par les patients durant leur période de contagiosité et répertoriés pendant l'enquête afin de retracer leurs contacts ont été extraits des dossiers des patients. Les périodes de contagiosité ont été évaluées pour les cas de tuberculose pulmonaire et de tuberculose laryngée et définies selon les lignes directrices provinciales et canadiennes $(13,14)$. La période de contagiosité se termine lorsque les patients sont isolés, procédure standard pour les cas contagieux.

\section{Analyse des données}

Des analyses descriptives ont été menées à l'aide du logiciel Excel (2010) (Microsoft Corp., Redmond, Washington). Les patients ont été répartis en deux groupes, selon qu'ils présentaient au moins un facteur de risque. Les proportions et moyennes non ajustées sont présentées. Dans le cas des 
patients ayant eu deux épisodes de tuberculose avec le même profil MIRU (trois cas), seul le premier épisode était inclus dans I'analyse descriptive étant donné que les données sur le second épisode étaient souvent manquantes.

Une analyse spatiale des endroits que les patients ont visités pendant leur période de contagiosité et de l'agrégation spatiale de ces endroits a été réalisée. Tous les endroits visités par les patients pendant qu'ils étaient contagieux ont été inclus dans cette analyse.

\section{Résultats}

Entre janvier 2003 et février 2016, 35 personnes ont correspondu à la définition de cas de cette éclosion dans trois régions contiguës du Québec : Montréal (21 cas), Montérégie (12 cas) et Laval (deux cas). De ce nombre, 29 cas confirmés présentaient le même génotype et six cas probables avaient un lien épidémiologique avec les cas présentant ce génotype. Trois cas avaient eu deux épisodes de tuberculose active avec le même profil MIRU, pour un nombre total de 38 épisodes. Deux de ces cas qui avaient eu un deuxième épisode étaient séropositifs pour le $\mathrm{VIH}$.

La figure 1 présente la courbe épidémique de cette éclosion. Bien qu'il n'y ait eu que huit cas en sept ans entre 2003 et 2009 le nombre de cas a triplé pour atteindre 27 entre 2010 et 2016.

Figure 1 : Éclosion de tuberculose à Montréal, Laval et en Montérégie entre 2003 et 2016

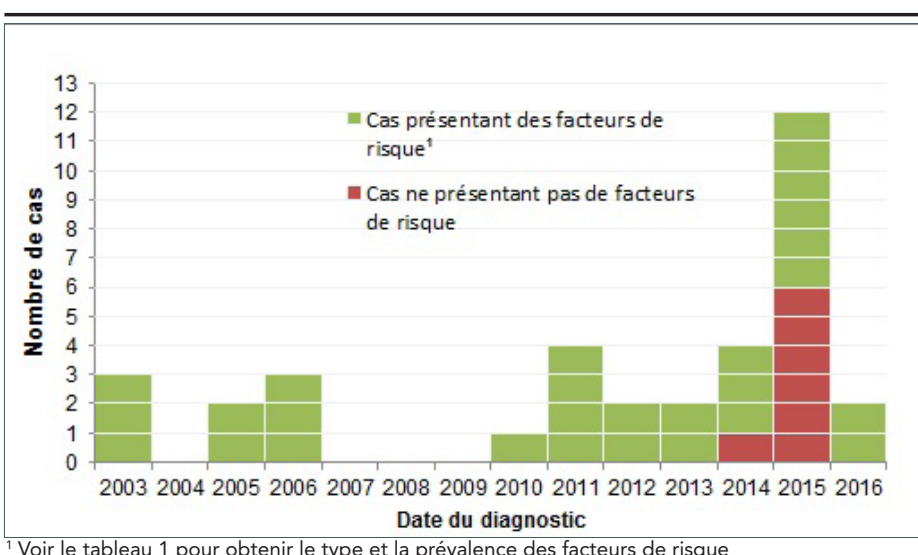

\section{Caractéristiques sociodémographiques}

L'âge des patients variait entre 1 et 69 ans (âge moyen : 37; écart interquartile : 33 à 47 ans), et $57 \%$ étaient des hommes. La plupart (30/35; $86 \%)$ étaient des non-Autochtones nés au Canada; trois (9\%) étaient des Inuits, un (3\%) était un Autochtone non-Inuit et un (3\%) était né à l'étranger.

\section{Facteurs de risque}

Les cas ont été classés en deux groupes : ceux qui présentaient des facteurs de risque $(n=28)$ et ceux qui ne présentaient aucun facteur de risque $(n=7)$. Les cas qui ne présentaient pas de facteur de risque étaient tous des contacts étroits (comme les membres de la famille) de deux des cas qui présentaient des facteurs de risque; parmi eux, quatre étaient des enfants de moins de 12 ans. Tous les cas (à l'exception d'un) qui ne présentaient aucun facteur de risque sont advenus en 2015 et représentaient la moitié des cas diagnostiqués cette année (figure 1).

Tous les cas avec facteurs de risque présentaient plus d'un facteur de risque. Le facteur de risque le plus courant était la consommation de drogues (93\%), plus précisément de cocaïne (54\%) et de crack (39\%). La proportion de données manquantes était élevée pour certains comportements à risque (comme I'utilisation de drogues injectables ou d'héroïne, le tabagisme et le travail du sexe) et pour les troubles de santé mentale (tableau 1).

Tableau 1 : Fréquence de facteurs de risque parmi les cas qui présentaient un ou plusieurs facteurs de risque ${ }^{1}$ $(n=28)$

\begin{tabular}{|c|c|c|c|}
\hline $\begin{array}{l}\text { Facteurs de } \\
\text { risque }\end{array}$ & $\begin{array}{c}\text { Nombre }(\%) \\
\text { de cas avec } \\
\text { ce facteur } \\
\text { de risque }\end{array}$ & $\begin{array}{l}\text { Nombre (\%) } \\
\text { de cas sans } \\
\text { ce facteur } \\
\text { de risque }\end{array}$ & $\begin{array}{l}\text { Nombre de } \\
\text { cas pour } \\
\text { lesquels } \\
\text { l'information } \\
\text { est } \\
\text { manquante }\end{array}$ \\
\hline $\begin{array}{l}\text { Consommation de } \\
\text { drogues }\end{array}$ & 26 (93\%) & $0(0 \%)$ & $2(7 \%)$ \\
\hline Cocaïne & $15(54 \%)$ & $3(11 \%)$ & $10(36 \%)$ \\
\hline Héroïne & $3(11 \%)$ & $5(18 \%)$ & 20 (71 \%) \\
\hline Crack & $11(39 \%)$ & 2 (7 \%) & 15 (54 \%) \\
\hline $\begin{array}{l}\text { Utilisation de } \\
\text { drogues injectables }\end{array}$ & $7(25 \%)$ & 5 (18\%) & 16 (57 \%) \\
\hline Tabagisme & $15(54 \%)$ & 1 (3 \%) & 12 (43 \%) \\
\hline $\begin{array}{l}\text { Consommation } \\
\text { abusive d'alcool }\end{array}$ & $18(64 \%)$ & 2 (7 \%) & $8(29 \%)$ \\
\hline Travail du sexe & $10(36 \%)$ & $6(21 \%)$ & 12 (43\%) \\
\hline $\begin{array}{l}\text { Troubles de santé } \\
\text { mentale }\end{array}$ & $8(29 \%)$ & 5 (18\%) & 15 (54 \%) \\
\hline $\begin{array}{l}\text { Co-infection hépatite } \\
\text { C }\end{array}$ & $12(43 \%)$ & 11 (39 \%) & $5(18 \%)$ \\
\hline Itinérance & $13(46 \%)$ & 15 (54 \%) & $0(0 \%)$ \\
\hline Co-infection VIH & $10(36 \%)$ & $14(50 \%)$ & $4(14 \%)$ \\
\hline $\begin{array}{l}\text { Immunosuppression } \\
\text { non-VIH }\end{array}$ & $5(18 \%)$ & 18 (64 \%) & $5(18 \%)$ \\
\hline $\begin{array}{l}\text { Antécédents } \\
\text { d'incarcération }\end{array}$ & $8(29 \%)$ & $12(43 \%)$ & $8(29 \%)$ \\
\hline
\end{tabular}

\section{Caractéristiques cliniques}

Le site de tuberculose active le plus fréquent était les poumons (30/35; $86 \%)$; cinq cas étaient atteints d'une tuberculose disséminée $(5 / 35 ; 14 \%)$. Trois patients sont décédés avant la fin du traitement, dont deux qui étaient séropositifs pour le $\mathrm{VIH}$. Les causes de décès étaient une surdose de drogue $(n=1)$, un cancer $(n=1)$ et une cause inconnue qui pourrait être liée à la tuberculose $(n=1)$. L'âge moyen des cas présentant des facteurs 
de risques était de 40 ans (étendue de 27 à 57 ans). Le tableau 2 présente la fréquence des autres caractéristiques cliniques selon la présence de facteurs de risque.

\section{Tableau 2 : Caractéristiques sociodémographiques et cliniques des cas selon la présence de facteurs de risque $^{1}$}

\begin{tabular}{|c|c|c|}
\hline Variables & $\begin{array}{c}\text { Nombre (\%) } \\
\text { de cas avec un } \\
\text { facteur de risque } \\
\text { et plus } \\
(n=28)\end{array}$ & $\begin{array}{l}\text { Nombre }(\%) \text { de } \\
\text { cas sans facteur } \\
\text { de risque }(n=7)\end{array}$ \\
\hline \multicolumn{3}{|l|}{ Sexe } \\
\hline Femmes & $12(43 \%)$ & $3(43 \%)$ \\
\hline Hommes & $16(57 \%)$ & $4(57 \%)$ \\
\hline \multicolumn{3}{|l|}{ Site d'infection ${ }^{2}$} \\
\hline Poumons & $24(86 \%)$ & $6(86 \%)$ \\
\hline Disséminée & $5(18 \%)$ & 0 \\
\hline Larynx & $1(4 \%)$ & 0 \\
\hline Autre & $3(11 \%)$ & $1(14 \%)$ \\
\hline Symptomatique & $26(93 \%)$ & $4(57 \%)$ \\
\hline \multicolumn{3}{|c|}{ Résultats de la radiographie des poumons } \\
\hline Normaux & $1(4 \%)$ & $1(14 \%)$ \\
\hline Anormale non cavitaire & $11(39 \%)$ & $5(71 \%)$ \\
\hline Lésions cavitaires & $12(43 \%)$ & $1(14 \%)$ \\
\hline \multicolumn{3}{|l|}{ Frottis d'expectorations } \\
\hline Négatifs & $2(7 \%)$ & $6(86 \%)$ \\
\hline Positivité (1+) & $6(21 \%)$ & 0 \\
\hline Positivité $(2+)$ & $5(18 \%)$ & 0 \\
\hline Positivité $(3+)$ & $4(14 \%)$ & $1(14 \%)$ \\
\hline Positivité (4+) & $6(21 \%)$ & 0 \\
\hline \multicolumn{3}{|l|}{ Évolution clinique } \\
\hline Guéris & $22(79 \%)$ & $7(100 \%)$ \\
\hline Encore en traitement & $2(7 \%)$ & 0 \\
\hline $\begin{array}{l}\text { Perdu de vue pendant le } \\
\text { suivi }\end{array}$ & $1(4 \%)$ & 0 \\
\hline Décédés & $3(11 \%)$ & 0 \\
\hline
\end{tabular}

Abréviations : $n$, nombre; \%, pourcentage

L'addition des nombres ne totalise pas $100 \%$ en raison de valeurs manquantes (moins de $20 \%$ pour toutes les variables)

${ }^{2}$ Les catégories d'infection ne s'excluent pas mutuellement étant donné que certains patients avaient plusieurs sites d'infection. Le ganglion lymphatique, la plèvre et l'abdomen figurent parmi les autres sites d'infection

Les cas présentant des facteurs de risque étaient presque tous symptomatiques (93\%), étaient atteints de la maladie à un stade avancé avec des cavités à la radiographie ( $43 \%$ ), étaient atteints de tuberculose disséminée (18\%) et avaient des frottis avec des taux élevés de positivité (de 3+ à 4+, 10/28; $35 \%$ ). Par conséquent, la durée moyenne de l'hospitalisation pour les cas présentant des facteurs de risque était de 38 jours (plage : de 0 à 571 jours) et la durée moyenne du traitement était de neuf mois (plage : de 6 à 32 mois). Pour les cas ne présentant aucun facteur de risque, la durée moyenne de l'hospitalisation était de 0 jour (plage : de 0 à 6 jours) et la durée moyenne du traitement était de six mois (plage : de 6 à 7 mois). La plupart des cas (29/35, $83 \%$ étaient guéris au terme du traitement. Aucune résistance aux antimicrobiens avant ou durant le traitement n'a été observée (y compris chez les cas sans facteur de risque). Chez les cas sans facteur de risque, le stade de la maladie semblait moins avancé. Parmi eux, trois cas étaient asymptomatiques et ont été diagnostiqués lors de la recherche des contacts.

\section{Intensité des interventions de santé publique}

Plusieurs facteurs ont entraîné une intensité élevée des interventions en santé publique. Dans certains cas, la recherche de soins s'est effectuée seulement lorsque les individus étaient à un stade avancé de la maladie, ce qui a entrainé une longue période de contagiosité avant le diagnostic et le traitement. Ceci a conduit à des interventions de recherche de contacts intensives et à la découverte de nouveaux cas. La période de contagiosité s'étendait de 0,5 à 18,9 mois (durée moyenne : 4 mois).

Des défis se sont aussi présentés au cours du traitement. En raison de difficultés à faire respecter l'observance au traitement médicamenteux et l'isolement, huit ordres de santé publique et huit ordres de cour ont été émis à neuf patients (26\%), dont la majorité présentaient des facteurs de risque (8/9; $89 \%)$. La fréquence des interventions par les infirmières en santé publique était élevée ou très élevée à l'égard de 22 cas avec facteurs de risque (79\%) et à l'égard d'un cas sans facteur de risque (14\%).

\section{Analyse spatiale}

Bien que les lieux fréquentés par les cas étaient répartis dans l'ensemble du territoire de Montréal, Laval et la Montérégie, certains endroits fréquentés étaient concentrés dans des points névralgiques. Les refuges, d'autres organisations non gouvernementales, ainsi que des lieux fréquentés pour le travail du sexe, le divertissement et la consommation de drogues se concentraient dans une zone particulière du centre-ville de Montréal qui comprend des fumeries de crack. Ces dernières avaient été visitées par plusieurs cas (carte non fournie à des fins de respect de la vie privée). Une analyse de l'agrégation spatiale a révélé deux principaux points névralgiques $(p<0,05)$ : un quartier défavorisé du centre-ville de Montréal et un milieu résidentiel en Montérégie.

\section{Discussion}

L'investigation de cette éclosion de cas de tuberculose montre qu'elle s'est produite au sein de populations urbaines marginalisées nées au Canada, en particulier chez les consommateurs de drogues et les sans-abri. Le principal facteur de risque est la consommation de drogues, en particulier de crack et de cocaïne. D'autres comorbidités, comme une co-infection VIH et les troubles de santé mentale, n'étaient pas rares. Un constat préoccupant était que l'éclosion s'est propagée à des contacts étroits qui ne présentaient aucun autre facteur de risque que cette exposition à la maladie.

Dans cette éclosion, le nombre de cas, la gravité de la maladie, les périodes de contagiosité, les comorbidités et l'évolution de la maladie étaient semblables à ceux des études antérieures menées à Montréal (de 1996 à 2007) et en Colombie-Britannique $(5,7)$. Le taux élevé de co-infection $\mathrm{VIH}$ pourrait expliquer, en partie, le stade avancé de la maladie active et le nombre élevé de cas au sein de ces populations, puisque la réactivation de I'infection tuberculeuse latente est plus susceptible d'être observée chez une population immunocompromise (14). Les dommages aux voies respiratoires causés par le fait de fumer 
du crack peuvent également contribuer à l'acquisition et à l'évolution de la maladie.

Un certain nombre de facteurs peuvent expliquer la transmission continue et la durée prolongée de cette éclosion (13 ans). Bien souvent, les sans-abri, les consommateurs de drogues illicites et les travailleurs du sexe n'ont pas de documents d'assurance-maladie, font l'objet de stigmatisation et ont des priorités liées aux besoins de base et à la consommation de drogues qui peuvent nuire à l'accès aux services de santé et aux services sociaux. Dans de tels cas, ces personnes peuvent ne pas accéder aux soins ou y accéder tardivement. En outre, comme la tuberculose active est relativement rare, les cliniciens pourraient ne pas envisager ce diagnostic chez les patients non migrants ou non-Autochtones. Cet éventail de facteurs peut également avoir été en cause dans d'autres éclosions de longue durée, comme une éclosion de 8 ans survenue en Colombie-Britannique (6) et une éclosion de 17 ans rapportée à Toronto (9). Dans le cadre d'un examen des caractéristiques des patients infectés à l'origine de 26 éclosions entre 2002 et 2011 , le profil de contagiosité élevée, les facteurs de risque sociaux (consommation d'alcool et de drogues, itinérance et incarcération) et un retard dans le diagnostic, semblables aux caractéristiques observées dans cette éclosion de Montréal, étaient très fréquentes (15). Malheureusement, la concomitance de plusieurs facteurs de risque et la longue durée du traitement étaient associés à une gestion des cas exigeante, à un niveau élevé d'interventions de santé publique et à un recours fréquent à des mesures juridiques. Une éclosion semblable qui a eu lieu à Toronto a également nécessité une gestion intensive des cas (4).

Notre analyse spatiale a confirmé les rapports précédents qui désignaient les sites de consommation de drogues comme un vecteur potentiel de transmission (7). Un secteur en particulier du centre-ville de Montréal est connu pour être un point névralgique pour la consommation de drogues avec la présence de plusieurs fumeries de crack (16). Ces endroits sont difficilement atteignables par un programme de dépistage de masse compte tenu de leur organisation, des règles et du contexte (17). Nous avons également localisé une zone périphérique en Montérégie, liée à un cas particulièrement contagieux, possiblement associé à un nouveau profil de consommation de substances que l'on retrouve principalement en milieu résidentiel (18). Les refuges ou les centres de réadaptation en dépendance ont aussi été visités par plusieurs cas au fil du temps, comme à Edmonton, où la transmission était manifestement associée à un petit nombre de refuges (8).

Il est difficile de concevoir et de mettre en œuvre un plan de contrôle de la tuberculose dans les populations urbaines marginalisées. La recherche de cas actifs dans les refuges et dans d'autres organismes communautaires entre 2013 et 2015 n'a pas permis de détecter de nouveaux cas, bien que d'autres cas reliés aient été rapportés plus tard. Des efforts concertés se poursuivent et incluent une analyse spatiale, un nouveau questionnaire sur le réseau social, des alertes de santé publique aux cliniciens et le dépistage systématique à certains endroits. La mobilité de cette population souligne l'importance de la coordination entre les régions, les organismes communautaires et les services de traitement de la toxicomanie.

Cette étude présente plusieurs limites. Tout d'abord, les données rétrospectives ont été utilisées pour décrire les facteurs de risque. Ces données sont recueillies de manière routinière mais non systématique. Le questionnaire d'enquête pour les cas a changé au fil du temps et variait d'une région à une autre. Par conséquent, les données manquantes étaient fréquentes. Deuxièmement, nous n'avons pas rendu compte de la recherche de contacts puisque ces renseignements étaient souvent difficiles à obtenir. Troisièmement, nous avons utilisé les lieux fréquentés pendant la période de contagiosité comme représentatifs des lieux d'acquisition. Aucun renseignement sur les endroits visités lorsque les cas ont contracté l'infection n'était disponible. Certains endroits potentiels de transmission étaient difficiles à obtenir et d'autres pourraient avoir été oubliés. Enfin, certains cas liés à l'éclosion peuvent avoir été omis, particulièrement les cas d'avant 2012, lorsque la MIRU-VNTR systématique des souches de tuberculose a débuté au Québec. Enfin, bien que le génotypage par MIRU-VNTR 24-loci permet une analyse hautement discriminante, dans de rares cas, les souches ayant le même profil MIRU peuvent ne pas être liées (19).

\section{Conclusion}

Bien que les migrants en provenance de pays où la maladie est endémique et les populations autochtones continuent de présenter un plus grand risque de contracter la tuberculose active au Canada, des éclosions de tuberculose liées à la consommation de drogues (en particulier de cocaïne et de crack) et à l'itinérance peuvent se produire dans des zones urbaines. Les populations des zones urbaines marginalisées qui consomment des drogues ont été identifiées comme un groupe de personnes à risque élevé ailleurs dans le monde. Cette éclosion indique qu'un indice de suspicion clinique élevé est nécessaire pour détecter la tuberculose au sein de cette population, en particulier si le VIH est présent.

\section{Remerciements}

Nous tenons à remercier tous les infirmiers et infirmières de santé publique des trois régions pour leur aide précieuse à la révision des cas et l'extraction des données. Nous tenons également à souligner la contribution du Laboratoire de santé publique du Québec et du personnel du Centre national de référence en mycobactériologie du Laboratoire national de microbiologie (Agence de la santé publique du Canada) pour la caractérisation des souches et le génotypage. Nous tenons à remercier André Bilodeau de la Direction régionale de santé publique du Centre intégré universitaire de santé et de services sociaux du Centre-Sud-de-l'île-de-Montréal pour son aide dans l'extraction des données et Robert Allard, du même établissement et Eric Levac de la Direction de santé publique du Centre intégré de santé et de services sociaux de la Montérégie-Centre de leur aide à la révision du présent article.

\section{Conflit d'intérêts}

Aucun. 


\section{Contributions}

JA - Conceptualisation, extraction et analyse des données, Rédaction - version originale, révision et édition, $C L$ Conceptualisation, enquête, extraction des données, Rédaction - révision et édition, $\mathrm{MB}$ - Conceptualisation, analyse spatiale, Rédaction - révision et édition, DMM - Conceptualisation, extraction des données, Rédaction - révision et édition, JLS Conceptualisation, enquête, extraction des données, Rédaction - révision et édition, EP - Conceptualisation, extraction des données, Rédaction - révision et édition, NT - Conceptualisation, enquête, NSi - Rédaction - révision et édition, NSa - Rédaction révision et édition, $\mathrm{PR}$ - Enquête, Rédaction - révision et édition, HS - Analyse en laboratoire, Rédaction - révision et édition, MMB - Conceptualisation, enquête, extraction des données, Rédaction version originale - révision et édition.

\section{Références}

1. Tuberculose. Aide-mémoire [Internet]. Genève: Organisation mondiale de la Santé; 2016 [consulté le 1 fév 2017]. Disponible à l'adresse: http://www.who.int/mediacentre/ factsheets/fs104/fr/.

2. Towards tuberculosis elimination: an action framework for low-incidence countries [Internet]. Geneva: World Health Organization; 2014 [consulté le 20 dec 2016]. 77p. Disponible à l'adresse : http://apps.who.int/iris/ bitstream/10665/132231/1/9789241507707_fra.pdf?ua=1.

3. La tuberculose au Canada 2014 : Prédiffusion [Internet]. Ottawa: Agence de la santé publique du Canada; 2016 [consulté le 20 déc 2016]. Disponible à l'adresse : http:// healthycanadians.gc.ca/publications/diseases-conditionsmaladies-affections/tuberculosis-2014-tuberculose/index-en. php\#a1.

4. Khan K, Rea E, McDermaid C, Stuart R, Chambers C, Wang J, Chan A, Gardam M, Jamieson F, Yang J, Hwang SW. Active tuberculosis among homeless persons, Toronto, Ontario, Canada, 1998-2007. Emerg Infect Dis. 2011;17(3):357-65. doi: 10.3201/eid1703.100833.

5. Gardy JL, Johnston JC, Ho Sui SJ, Cook VJ, Shah L, Brodkin E, Rempel S, Moore R, Zhao Y, Holt R, Varhol R, Birol I, Lem M, Sharma MK, Elwood K, Jones SJ, Brinkman FS, Brunham RC, Tang P. Whole-genome sequencing and socialnetwork analysis of a tuberculosis outbreak. N Engl J Med. 2011;364(8):730-9. doi: 10.1056/NEJMoa1003176.

6. Cheng JM, Hiscoe L, Pollock SL, Hasselback P, Gardy JL, Parker R. A clonal outbreak of tuberculosis in a homeless population in the interior of British Columbia, Canada, 20082015. Epidemiol Infect. 2015;143(15):3220-6.

7. Tan de Bibiana J, Rossi C, Rivest $P$, Zwerling A, Thibert L, Mclntosh F, Behr MA, Menzies D, Schwartzman K. Tuberculosis and homelessness in Montreal: a retrospective cohort study. BMC Public Health. 2011;11:833. doi: 10.1186/1471-2458-11-833.

8. Moreau D, Gratrix J, Kunimoto D, Beckon A, Der E, Hansen E, Chui L, Ahmed R. A shelter-associated tuberculosis outbreak: a novel strain introduced through foreign-born populations. Can J Public Health. 2012;103(6):e408-12.

9. Mehaffy C, Guthrie JL, Alexander DC, Stuart R, Rea $\mathrm{E}$, Jamieson FB. Marked microevolution of a unique Mycobacterium tuberculosis strain in 17 years of ongoing transmission in a high risk population. PLoS One. 2014;9(11):e112928. doi: 10.1371/journal.pone.0112928.

10. Bamrah S, Yelk Woodruff RS, Powell K, Ghosh S, Kammerer JS, Haddad MB. Tuberculosis among the homeless, United States, 1994-2010. Int J Tuberc Lung Dis. 2013;17(11):14149. doi: 10.5588/ijtld.13.0270.

11. McAdam JM, Bucher SJ, Brickner PW, Vincent RL, Lascher $\mathrm{S}$. Latent tuberculosis and active tuberculosis disease rates among the homeless, New York, New York, USA, 19922006. Emerg Infect Dis. 2009; 15(7):1109-11. doi: 10.3201/ eid1507.080410.

12. Rivest $P$, Street MC. Épidémiologie de la tuberculose au Québec de 2008 à 2011 [Internet]. Québec: Agence de la santé et des services sociaux; 2014 [consulté le 20 déc 2016]. Disponible à l'adresse : http://publications.msss.gouv. qc.ca/msss/fichiers/2014/14-266-01W.pdf.

13. Rivest $P$, Abdelaziz $N$, Déry $S$, Isler $M$, Lacroix $C$, Thibert L. Guide d'intervention pour la tuberculose - Édition 2012 [Internet]. Québec: Ministère de la santé et des services sociaux; 2013 [consulté le 20 déc 2016]. Disponible à l'adresse : http://publications.msss.gouv.qc.ca/msss/ fichiers/2012/12-271-01W.pdf.

14. Normes canadienne pour la lutte antituberculeuse $7^{\mathrm{e}}$ édition. Ottawa: Agence santé publique du Canada; 2014. Disponible à l'adresse : http://www.phac-aspc.gc.ca/tbpclatb/pubs/tb-canada-7/index-fra.php.

15. Haddad MB, Mitruka K, Oeltmann JE, Johns EB, Navin TR. Characteristics of tuberculosis cases that started outbreaks in the United States, 2002-2011. Emerg Infect Dis. 2015;21(3):508-10.

16. Leclerc $P$, Fall A, Morissette $C$. Estimation de la taille et caractérisation de la population utilisatrice de drogues par injection à Montréal. Direction de santé publique. Montréal: Agence de la santé et des services sociaux de Montréal; 2013. p. 4.

17. Roy E, Arruda N. Exploration of a crack use setting and its impact on drug users' risky drug use and sexual behaviors: the case of piaules in a Montreal neighborhood. Subst Use Misuse 2015;50(5):630-41.

18. Milot DM. Évaluation du besoin et de la pertinence de l'implantation d'un service d'injection supervisée en Montérégie [thèse de maîtrise]. Montréal: Université de Montréal; 2014. Disponible à l'adresse : https://papyrus.bib. umontreal.ca/xmlui/handle/1866/11821.

19. Christianson S, Wolfe J, Orr P, Karlowsky J, Levett PN, Horsman GB, Thibert L, Tang P, Sharma MK. Evaluation of 24 locus MIRU-VNTR genotyping of Mycobacterium tuberculosis isolates in Canada. Tuberculosis (Edinb). 2010;90(1):31-8. 